\title{
Development and classification of rubeosis iridis in diabetic eye disease
}

\author{
LEILA LAATIKAINEN \\ From the Department of Ophthalmology, University of Helsinki, Finland
}

SUMmARY The evolution and characteristics of diabetic rubeosis were studied in 33 eyes, and the following vascular abnormalities were found: (1) Dilated leaking capillaries around the pupil; (2) irregular or slow filling of the radial arteries; (3) superficial arborising newly formed vessels, usually starting in the chamber angle; and (4) dilatation and leakage of the radial vessels either before or after the development of neovascular glaucoma. None of the vascular changes was specific for diabetes. Four grades of diabetic iridopathy were distinguished on the extent of rubeosis: I, peripupillary vessel dilatations with leakage; II, early neovascularisation mainly in the chamber angle; III, prominent rubeosis with or without neovascular glaucoma; and IV, florid rubeosis.

Abnormal vessels in the iris in diabetic patients were first observed by Salus (1928), who called the condition rubeosis iridis. Neovascularisation of the iris is often followed by neovascular glaucoma, with its associated blindness and pain. During the last few years more attention has been paid to diabetic rubeosis, because neovascular glaucoma is one of the commonest postoperative complications in diabetic patients after vitrectomy (Michels, 1978).

In this paper the characteristic vascular changes of the iris in diabetic eyes are described, and a classification of diabetic rubeosis is presented.

\section{Patients and methods}

Thirty-three eyes with varying severity of diabetic iridopathy were studied by fluorescein angiography of the iris. In many of them several angiographies were performed during the course of the disease at a few months' interval. Each of the patients received thorough ophthalmological examination, including testing of visual acuity, tonometry, biomicroscopy, gonioscopy, and ophthalmoscopy. In most eyes fundus photography and fluorescein angiography were also performed.

In iris fluorescin angiography the technique described in detail by Vannas (1969) was used. A Zeiss-Opton slit lamp coupled with an automatic Robot camera with the filter combination of a Baird Atomic B4 and a Schott GG14 $3 \mathrm{~mm}$ and the

Address for reprints: Leila Laatikainen, MD, University Eye Hospital, Haartmaninkatu 4 C, 00290 Helsinki 29, Finland film Kodak Tri-X Pan were used. The photography was begun 10 seconds after the injection of $5 \mathrm{ml}$ of $10 \%$ sodium fluorescein (Fluorescite) into the antecubital vein. The pictures were taken at about 2-second intervals.

\section{Results}

The following types of vascular abnormalities in the iris were revealed by fluorescein angiography: (1) Dilated leaking vessels around the pupil; (2) irregular or slow filling of the radial arteries; (3) superficial arborising neovascularisation, usually starting in the chamber angle; and (4) dilatation and leakage of the radial vessels, particularly arteries.

On the basis of the angiographic findings 4 grades of diabetic iridopathy were separated.

Grade I. Peripupillary vessel dilatations with leakage. Dilated leaking capillaries around the pupil were the first abnormalities found on the iris angiograms (Fig. 1). Irregularities in the filling of the radial vessels were seen (Fig. 1a), but no avascular areas were present. There was no leakage from the radial vessels, but dilated peripupillary capillaries leaked profusely (Fig. $1 b$ ).

Peripupillary vessel dilatations were either uniocular or more usually binocular. Most of these vessels were also seen by biomicroscopy, particularly in red-free light. Peripupillary changes were also seen in eyes with fairly mild retinopathy, and they have been found to remain unchanged for several years.

Grade II. Early neovascularisation mainly in the 
Fig. 1 (a, b) Fluorescein angiogram of the right iris. Irregular filling of radial arteries and dilated vessels around the pupil (a). Marked leakage from peripupillary vessels (b)
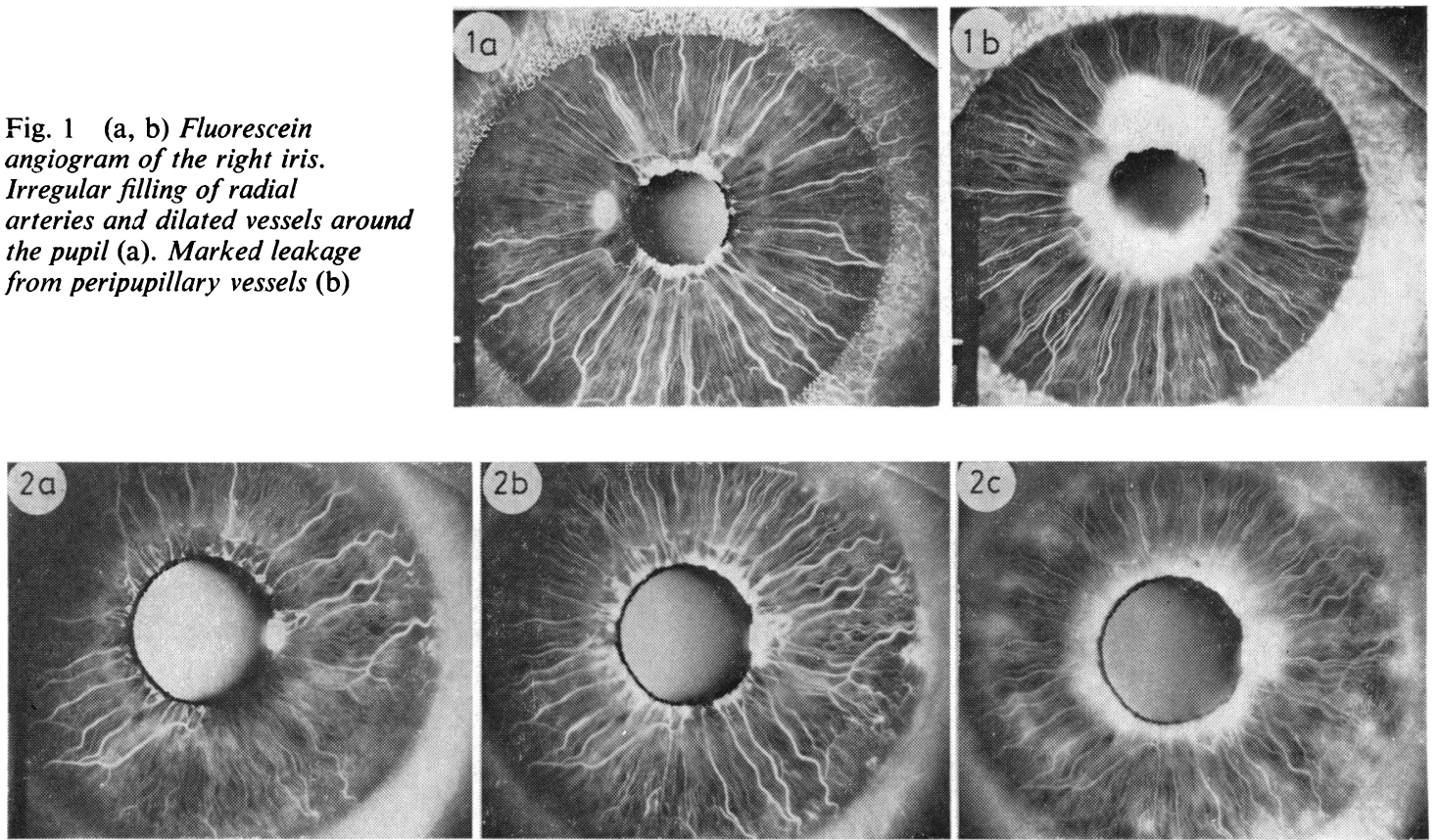

Fig. 2 (a-c) Fluorescein angiogram of the left iris. Irregular filling of radial arteries and dilated vessels around the pupil (a). Early neovascularisation appearing from the angle (b). Leakage around the pupil from the new vessels and radial arteries $(\mathrm{c})$

chamber angle. In addition to peripupillary vessel dilatations small irregular arborising superficial newly formed vessels appeared in the chamber angle (Figs. 2 and $3 a, b$ ) and less commonly elsewhere on the iris. These vessels filled in the early arterial phase (Figs. $2 a$ and $3 a$ ) and leaked fluorescein (Figs. $2 c$ and $3 b$ ). Because of their localisation in the angle, the first new vessels were often seen earlier by gonioscopy than on angiograms. Filling of the radial arteries was often irregular (Fig. $2 a$ ) or slow in all arteries (Fig. 3), and some localised leakage was seen from the radial vessels (Fig. $2 c$ ).

At this stage most of the angle remained open and the intraocular pressure was normal. In 3 eyes, however, neovascular glaucoma developed before progression of rubeosis further along the iris (Fig. $4 a, b)$. In spite of successful trabeculectomy, rubeosis continued its progression in a few months to grade III (Figs. $4 c, d$ ). All eyes with definite new vessels in the iris also had extensive vascular closure in the retina, and neovascularisation was also found in the fundus either preretinally or on the optic disc.

Grade III. Prominent rubeosis with or without neovascular glaucoma. Arborising new vessels were more prominent and had grown out of the angle covering more of the iris surface (Figs. $3 c-f, 4 c, d$, and 5). New vessels filled in the early arterial phase
(Fig. 3c). In some eyes filling of the radial vessels had further slowed down with the progression of rubeosis, and radial arteries were dilated and leaked fluorescein (Figs. 3e,f). Thus generalised marked leakage from the main radial vessels was found to precede (Fig. $3 f$ ) or coincide with (Fig. 4b) neovascular glaucoma. After normalisation of the intraocular pressure by surgery leakage from the radial vessels decreased (Fig. $4 d$ ). In most eyes with prominent rubeosis, however, radial vessels seemed to be less damaged, and only mild leakage of fluorescein was found (Fig. 5).

Most eyes with grade II or III rubeosis had dilated vessels around the pupil (Figs. 3 and 4), but definite new vessels were much less common and less prominent around the pupil than in the chamber angle. Even in some eyes with early neovascular glaucoma there were no certain new vessels except those in the angle (Fig. 4a). Mild eversion of the pigmented border or slight deformity of the pupil was, however, often noticed.

In the majority of eyes which developed grade III rubeosis and neovascular glaucoma progression of retinal vascular closure had been extremely rapid, preceding development of rubeosis by only a few months.

Grade IV. Florid rubeosis. In the most advanced 

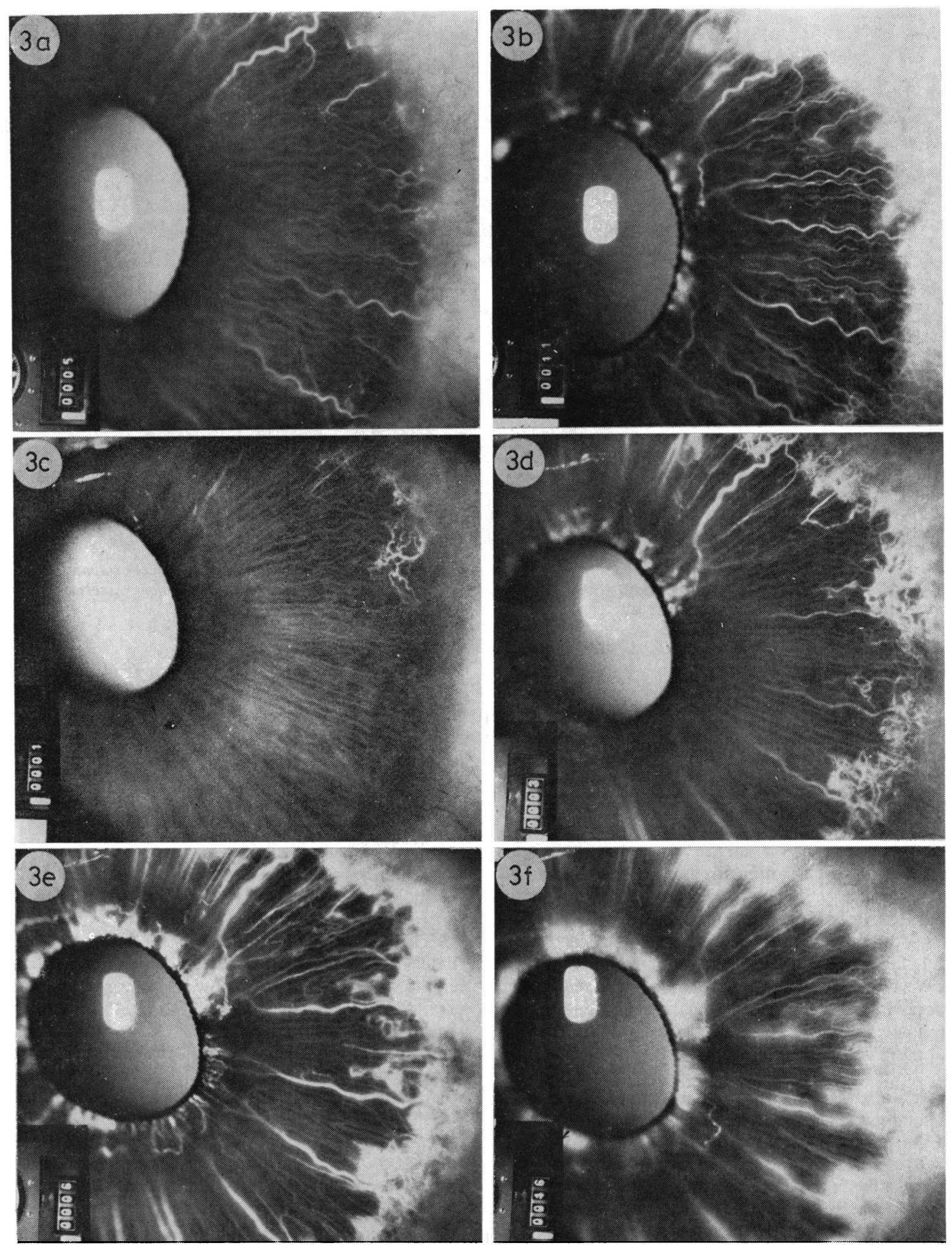

Fig. 3 (a, b) Fluorescein angiogram of the left iris. Slow filling of radial arteries (about 5 seconds from the iris root to the pupillary border) (a). Dilated vessels around the pupil and early neovascularisation in the angle with leakage (b). (c-f) Same iris 6 months later. Early filling of new vessels in the angle (c, d). Radial arteries and peripupillary vessels dilated with leakage (e, f). IOP $15 \mathrm{mmHg}$. Neovascular glaucoma developed a month later 
Fig. 4 (a, b) Fluorescein angiogram of the right iris after recognition of neovascular glaucoma. Areas of peripheral neovascularisation coming from the angle (a) with leakage (b). Leakage from the radial arteries and dilated capillaries around the pupil (b). (c-d) Same iris 10 months later after trabeculectomy showing considerable progression of rubeosis from the angle and more dilated vessels around the pupil (c) with leakage (d), but midstromal portion almost free of neovascularisation. IOP $19 \mathrm{mmHg}$
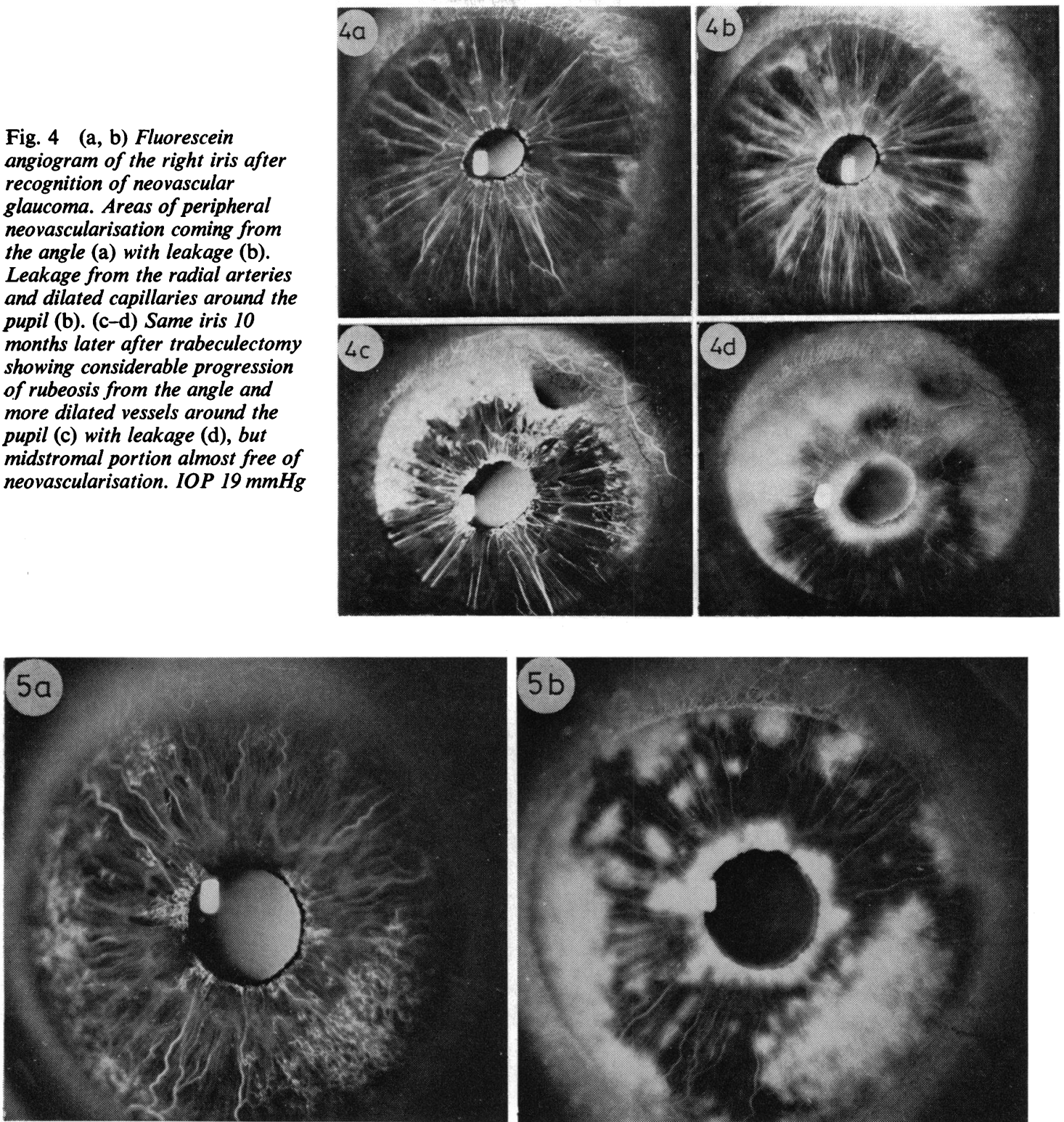

Fig. 5 (a, b) Fluorescein angiogram of the right iris. Considerable rubeosis mainly in the periphery of the iris with profuse leakage. Mild leakage from the radial vessels

stages of rubeosis new vessels covered the whole of the iris, the result being complete angle closure, neovascular glaucoma, and further eversion of the pigmented border of the pupil (Figs. $6 a, b$ ). There was extensive leakage from the new vessels all over the iris.

In longstanding cases new vessels were found to cross the pupillary border (Fig. $7 a, b$ ), but they were smaller in size and leaked less than in the earlier active stages of the disease.

\section{Discussion}

The first vascular abnormalities in the iris in diabetic eye disease are dilated leaking vessels around the pupil (Jensen and Lundbaek, 1968; 

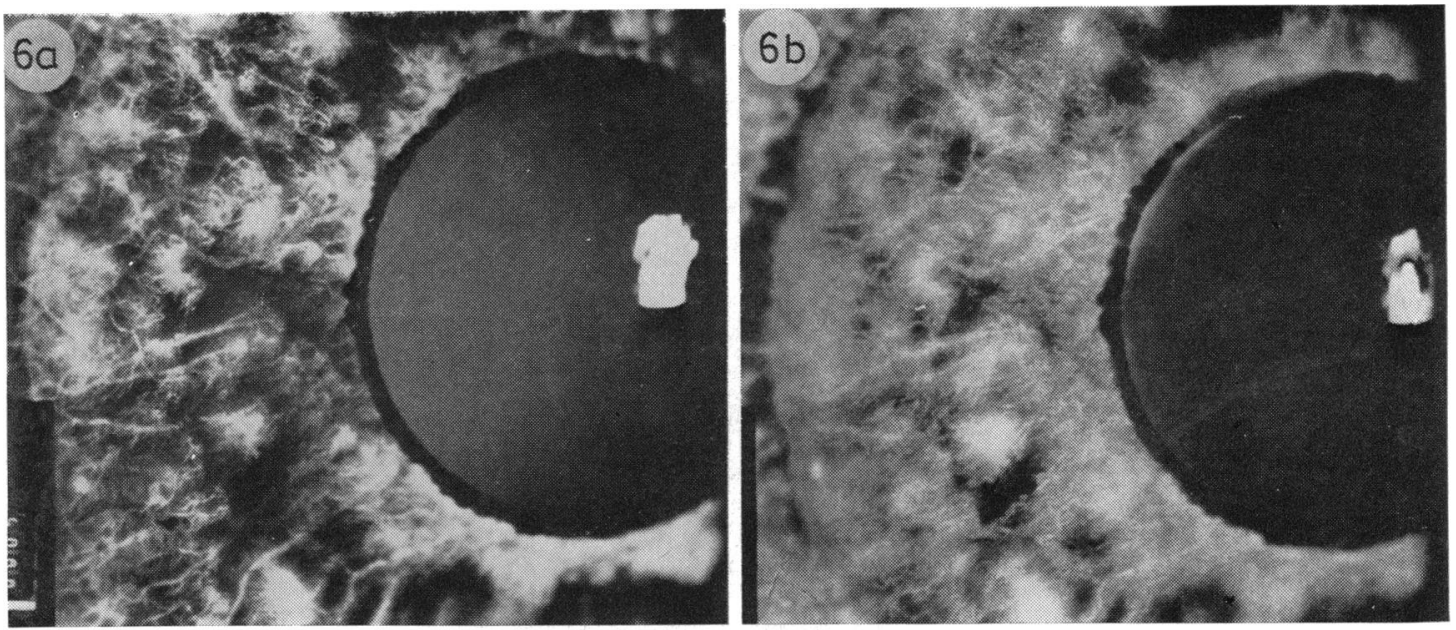

Fig. 6 (a, b) Fluorescein angiogram of the right iris. Extensive aborising neovascularisation coming from the angle covering the whole iris (a) with marked leakage (b). IOP $40 \mathrm{mmHg}$

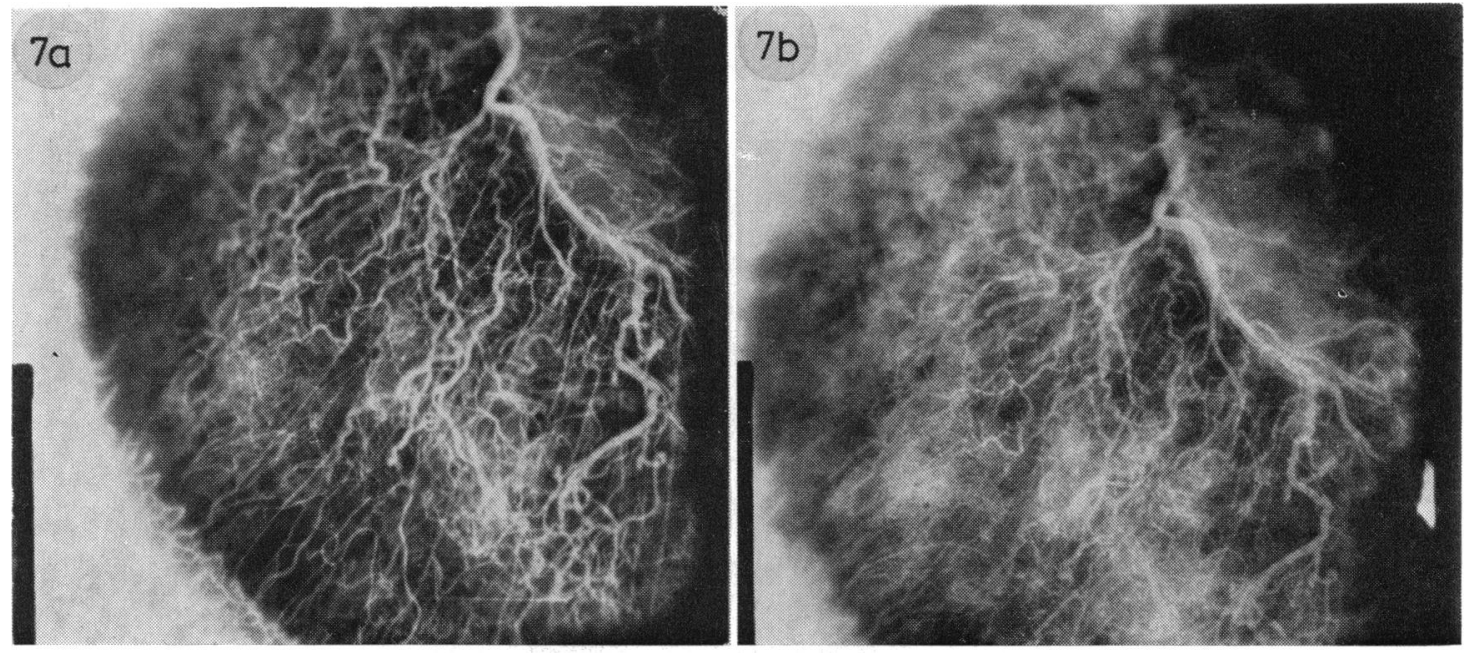

Fig. 7 (a, b) Iris fuorescein angiogram of blind normotensive eye 19 months after iridencleisis. A large vessel trunk at the pupillary margin with branches both to the iris and the cataractous lens (a) with mild leakage (b)

Baggesen, 1969; Friedburg et al., 1973). In healthy eyes no fluorescein leakage is present, because the endothelial cells of the vessels of the human iris lack fenestrae and seem to be joined by tight junctions (Hogan et al., 1971). Peripupillary vessel dilatations, however, are not specific for diabetes. Peripupillary vessel tufts have also been described as an isolated finding (Sellman, 1972; Israel and Lorenzetti, 1974) or in association with other diseases like myotonic dystrophy (Cobb et al., 1970) or congenital cyanotic heart disease (Krarup, 1977). More generalised dilatation and leakage of the peripupillary capillaries are also commonly seen in chronic glaucoma (Vannas, 1969) and in central retinal vein occlusion (Laatikainen and Blach, 1977).

In this study it appeared that most of the early peripupillary vessel abnormalities in diabetics were dilated pre-existing capillaries that leaked fluorescein rather than newly formed vessels. Peripupillary vessel dilatations were often associated with irregular filling of the radial arteries. These changes were found to occur also in eyes with fairly mild retinopathy without significant retinal vascular occlusion. Therefore peripupillary vessel dilatations do not 
usually indicate an immediate danger for neovascular glaucoma, which occurs in relation to severe retinopathy.

The other vascular changes or types of neovascularisation were not specific for diabetic eye disease, for similar changes are seen associated with other vascular diseases. In the majority of eyes definite neovascularisation started in the chamber angle, and therefore newly formed vessels were usually seen earlier by gonioscopy than by fluorescein angiography. Only occasionally was neovascularisation found to spread from the pupillary border towards the periphery of the iris.

Together with new vessel formation, radial vessels, particularly arteries, started to leak fluorescein. Similar leakage has been found in the ischaemic type of central retinal vein occlusion (Vannas and Raitta, 1972; Cappin and Whitelocke, 1974; Laatikainen and Blach, 1977) and in a recent central retinal artery occlusion (Kottow, 1976), indicating the possible effect of posterior segment ischaemia on the iris. Areas of vascular closure comparable to those in the retina were not seen in the iris in any of the patients, although in some eyes filling of the radial arteries was slow. This is in contrast with the findings of Baggesen (1969), who found that the time between the appearance of fluorescence in the annulus major and at the pupillary border was shorter in diabetics with rubeosis than in nondiabetics. In eyes with high intraocular pressure or soon after a glaucoma attack leakage of the radial vessels was apparently due to local ischaemia of the iris. Similar leakage has also been described after an attack of primary angle-closure glaucoma (Vannas, 1969). Whether both posterior and anterior segment ischaemia are needed for the development of rubeosis in diabetics remains unknown.

In this series development of neovascular glaucoma was always associated with extensive and rapidly progressing retinal vascular closure. It is well known that many diabetics with retinal vascular closure and proliferative retinopathy do not, however, develop neovascular glaucoma (Madsen, 1971a). In most diabetics development of occlusive retinopathy is gradual and slow, resulting in progressive atrophy of the retina, which, it seems, does not stimulate new vessel formation as the oedematous hypoxic retina does. This might be the reason for the great difference in the incidence and rapidity of development of rubeosis and neovascular glaucoma in diabetics with proliferative retinopathy and in patients with the ischaemic type of central retinal vein occlusion. In the latter disease one-third of the eyes develop neovascular glaucoma (Laatikainen and Kohner, 1976). It is possible that a similar incidence would be found in diabetics if only the rapidly progressing cases of occlusive retinopathy were included.

Spontaneous disappearance of iris neovascularisation in diabetics has been reported (Ohrt, 1967; Madsen, 1971a, 1971b). In this study it was not seen in any of the untreated eyes. In 2 eyes panretinal photocoagulation was performed after the discovery of iris neovascularisation. In 1 eye progression of rubeosis seemed to be arrested and in the other eye rubeosis disappeared after photocoagulation, supporting the earlier observations of the favourable effect of panretinal photocoagulation on rubeosis iridis (Little et al., 1976; Laatikainen, 1977).

Photographs and fluorescein angiograms were taken by Mr Erkki Manninen and Mr Seppo Lemberg.

This work was supported by the Paulo Foundation.

\section{References}

Baggesen, L. H. (1969). Fluorescence angiography of the iris in diabetics and non-diabetics. Acta Ophthalmologica, 47, 449-460.

Cappin, J. M., and Whitelocke, R. (1974). The iris in central retinal vein thrombosis. Proceedings of the Royal Society of Medicine, 67, 1048-1051.

Cobb, B., Shilling, J. S., and Chisholm, I. H. (1970). Vascular tufts at the pupillary margin in myotonic dystrophy. American Journal of Ophthalmology, 69, 573-582.

Friedburg, D., Wigger, H., and Schultheiss, K. (1973). Fluoreszenzangiographie der Iris bei Diabetikern. Klinische Monatsblätter für Augenheilkunde, 162, 218-223.

Hogan, M. J., Alvarado, J. A., and Weddell, J. E. (1971). Histology of the Eye, pp. 236-240. Saunders: Philadelphia.

Israel, M. P., and Lorenzetti, D. W. C. (1974). Bilateral microhemangiomas of the pupillary border with later hyphema. Canadian Journal of Ophthalmology, 9, 138-140.

Jensen, V. A., and Lundbaek, K. (1968). Fluorescence angiography of the iris in recent and long-term diabetes. Preliminary communication. Acta Ophthalmologica, 46, 584-585.

Kottow, M. (1976). Iris angiography in vascular diseases of the fundus. Documenta Ophthalmologica, Proceedings Series No. 9, 465-471.

Krarup, J. C. (1977). Atypical rubeosis iridis in congenital cyanotic heart disease. Report of a case with microhaemangiomas at the pupillary margin causing spontaneous hyphaemas. Acta Ophthalmologica, 55, 581-585.

Laatikainen, L. (1977). Preliminary report on effect of retinal panphotocoagulation on rubeosis iridis and neovascular glaucoma. British Journal of Ophthalmology, 61, 278-284.

Laatikainen, L., and Blach, R. K. (1977). Behaviour of the iris vasculature in central retinal vein occlusion. A fluorescein angiographic study of the vascular response of the retina and the iris. British Journal of Ophthalmology, 61, 272-277.

Laatikainen, L., and Kohner, E. M. (1976). Fluorescein angiography and its prognostic significance in central retinal vein occlusion. British Journal of Ophthalmology, 60, 411-418.

Little, H. L., Rosenthal, A. R., Dellaporta, A , and Jacobson, D. R. (1976). The effect of pan-retinal photocoagulation on rubeosis iridis. American Journal of Ophthalmology, 81, 804-809. 
Madsen, P. H. (1971a). Rubeosis of the iris and haemorrhagic glaucoma in patients with proliferative diabetic retinopathy. British Journal of Ophthalmology, 55, 368-371.

Madsen, P. H. (1971b). Haemorrhagic glaucoma. Comparative study in diabetic and nondiabetic patients. British Journal of Ophthalmology, 55, 444-450.

Michels, R. G. (1978). Vitrectomy for complications of diabetic retinopathy. Archives of Ophthalmology, 96, 237246.

Ohrt, V. (1967). Diabetic iridopathy. Clinical studies of the pigment layer of the iris, pupillary function and rubeosis iridis in diabetic patients. Diss. Univ. Aarhus.
Salus, R. (1928). Rubeosis iridis diabetica, eine bisher unbekannte diabetische Irisveränderung. Medizinische Klinik, 24, 256-258.

Sellman, A. (1972). Hyphaema from microhaemangiomas. Acta Ophthalmologica, 50, 58-61.

Vannas, A. (1969). Fluorescein angiography of the vessels of the iris in pseudoexfoliation of the lens capsule, capsular glaucoma and some other forms of glaucoma. Acta Ophthalmologica, Suppl. 105.

Vannas, S., and Raitta, C. (1972). Microcirculatory disturbances of occlusive diseases of the eye. Documenta Ophthalmologica, 33/2, 345-366. 